\title{
STUDIES ON HUMAN SCHISTOSOMIASIS: CHEMOTHERAPY, IMMUNITY AND MORBIDITY
}

\author{
A. E. BUTTERWORTH
}

\begin{abstract}
SUMMARY
At present, chemotherapy is the mainstay of schistosomiasis control programmes, but the most appropriate method of drug delivery frequently remains uncertain. In a six-year study in Machakos District, Kenya, designed to compare different drug delivery protocols, we have found that treatment only of infected primary school children is an effective, inexpensive and logistically practicable method of achieving a long-term reduction in intensity of infection in an area of high transmission but low morbidity. However, it is argued that even this simple approach to drug delivery is still not ideal-especially in areas of high morbidity in which intensity of infection may not be the only determinant of severe disease-and that a long-term approach to alternative control measures, especially vaccination, is desirable. Extensive studies have
\end{abstract}

shown that a range of experimental hosts can be substantially protected against a challenge infection by immunisation not only with live attenuated larvae but also with individual recombinant antigens. The demonstration that an age-dependent acquired resistance to reinfection after chemotherapy also occurs in man offers encouragement that immunisation should theoretically be feasible: and attempts have been made to relate an observed resistance to reinfection in older individuals to various immune responses both to whole parasite antigens and to individual recombinant peptides. However, many questions remain unanswered, especially in the context of understanding what factors, other than intensity of infection, contribute to the development of severe morbidity in particular geographical foci, and some possibilities are discussed.

\section{RÉSUMÉ : Schistosomiase humaine : chimiothérapie, immunité et morbidité.}

La chimiothérapie est pour l'instant la méthode de base utilisée par les programmes de lutte contre la bilharziose, mais la façon la plus appropriée pour la distribution des médicaments reste débattue. Une étude de six années effectuée dans le district du Machakos, au Kenya, a eu pour objectif de comparer différents protocoles de chimiothérapie. Au terme de cette étude, nous avons conclu que le traitement seulement des enfants infectés de 5 à 10 ans est efficace, peu coûteux, et facile à mettre en œuvre, pour aboutir à une diminution de l'intensité de l'infection dans des zones de haute transmission mais de faible morbidité. Cependant, on pourrait argumenter que cette approche simple n'est pas idéale spécialement dans les zones de forte morbidité où l'intensité de l'infection n'est peut-être pas le seul paramètre conduisant à une forme sévère de la maladie et qu'une méthode alternative de contrôle à long terme, spécialement la vaccination, est souhai- table. De nombreux travaux ont montré que différents animaux de laboratoire pouvaient être protégés contre l'infection par l'immunisation, non seulement avec des parasites vivants atténués mais aussi par des antigènes recombinants même pris isolément. La démonstration que la résistance acquise à la réinfection après chimiothérapie, qui est âge dependante, existe aussi chez l'homme, laisse sérieusement envisager que la vaccination est théoriquement possible. Dans cette perspective, nous avons tenté de corréler la résistance observée à la réinfection chez des individus plus âgés à des paramètres immunologiques variés, à la fois contre des antigènes totaux ou recombinants. Cependant, de nombreuses questions restent encore posées, en particulier celle de comprendre quels sont, en dehors de l'intensité de l'infection, les autres paramètres qui conduisent au développement d'une morbidité sévère dans des zones géographiques précises. Quelques hypothèses sont discutées.

\section{INTRODUCTION}

A distinctive property of the schistosomes is that, as is the case of other helminths in their definitive hosts, the adult worms do not divide, and each female worm lays a limited number of eggs in any given time period. In the case of Schistosoma mansoni, the development of the severe manifestations of hepatosplenic disease depends at least in

Molteno Laboratory of Parasitology, Department of Pathology, University of Cambridge, Tennis Court Road, Cambridge CB2 1QP. part on the numbers of eggs deposited in the tissues. It follows that the development of disease also depends on the actual numbers of adult worms, and the length of time that they are present-that is, on the intensity and duration, rather than simply the presence, of infection. The distribution of intensity of infection in a population, as reflected by egg excretion in the faeces, is highly overdispersed (Anderson, 1987): a small number of individuals harbour most of the worms, and these are the ones who will go on to develop severe disease. Therefore, for a control measure to be useful in reducing morbidity, it does not necessarily have to be totally effective in preventing transmission or infection: any measure that even partially 
reduces the establishment of adult worms may have a great impact on morbidity, by markedly reducing the numbers of individuals in the high intensity category.

Within this framework, the present paper discusses two approaches to the control of schistosomiasis:

- first, chemotherapy, as the most effective measure currently available;

- and secondly, vaccination, as a possible measure for the future;

in the context of laboratory and field studies on Schistosoma mansoni infections carried out over the past ten years in collaboration with the Kenya Ministry of Health and the Kenya Medical Research Institute.

\section{DELIVERY OF CHEMOTHERAPY}

Of the various methods of control that are theoretically possible, the most widely favoured at the moment is chemotherapy, especially since the introduction about fifteen years ago of two drugs, oxamniquine and praziquantel, that are both effective and safe. However, it is by no means obvious how chemotherapy should be delivered. One technique is by mass chemotherapy programmes that involve the treatment of all infected individuals in a community. This method is effective, but is also expensive in terms of both drug costs and the use of skilled labour, and an alternative that has been proposed and tested in the past has been to treat all heavily-infected individuals, on the grounds that they are the ones who will go on to develop severe disease. This approach leads to a saving in drug costs, but still suffers from the problem that examination and treatment must be carried out on a community-wide basis. Therefore, in a recently-completed six year study (Butterworth et al., 1989, and in preparation), we have tested an alternative approach, involving treatment, with retreatment as necessary, only of infected primary school children, on the grounds that they are the ones:

- who will include most of the heavily infected individuals in a community;

- who will go on to develop severe morbidity;

- who are most likely to become rapidly reinfected after treatment, because of either high exposure or lack of immunity or both;

- who contribute most to transmission, by indiscriminate defaecation in and around the waterbodies;

- and who are most easily, cheaply and rapidly accessible, for both diagnosis and treatment, through the framework of the primary schools.

Three areas were established in which these approaches were compared, together with a fourth, witness area in which treatment was offered, for ethical reasons, only to those with very heavy infections of more than 800 eggs per gram. Each of these areas, which were in the same general region of Kangundo, in Machakos District in Kenya, was surrounded by a buffer zone or cordon sanitaire in which the same treatment was administered, but which was not otherwise followed-up. In Area A, treatment was offered to all infected individuals in October 1983, and again in April 1985. In the other areas, a year of precontrol observations was carried out before treatment in 1985, either of infected schoolchildren in Area S, or of those excreting more than 100 eggs per gram in Area $\mathrm{H}$, or of those excreting more than 800 eggs per gram in the witness area, Area W. The various areas were then followed through to April 1989.

Area A served as a positive control. At the initial round of treatment in October 1983, a surrounding buffer zone was not included. Transmission therefore continued, through residents of adjacent areas, and was associated with some reinfection during the following year. Retreatment in 1985, both in Area A and in the surrounding buffer zone, was followed by three years of very low intensities of infection: such reinfection as did occur was most marked in young children aged 9 to 12 . Although pretreatment intensities in Area A lietune were lower than in the other areas, the conclusion could be drawn that treatment of all infected individuals was a highly effective method of delivering chemotherapy.

In Area $\mathrm{H}$, pretreatment intensities in two surveys were considerably higher than in Area A. Treatment was given in April 1985 to those excreting more than 100 eggs per gram: this had relatively little effect on prevalence, but caused a marked and persistent reduction in mean intensity. Again, reinfection was most marked among the 9 to 12 year olds, as reflected by the distribution by age both of geometric mean intensity of infection and of the prevalence of heavy infections.

In the witness area, Area $\mathrm{W}$, treatment of those few individuals with very heavy infections had little effect on prevalence, but a surprisingly marked immediate impact on intensity. However, reinfection occurred rapidly, although with yearly fluctuations, and was again most marked in children.

In the test area, Area S, in which only schoolchildren were treated, there was again little effect on prevalence, but a dramatic decline in intensity, especially among the schoolchildren who had been treated. Some reinfection did occur among these children over the subsequent three years, but overall intensities remained low. In addition, although the initial prevalence of clinical morbidity in pretreatment surveys was relatively low (7 to $17 \%$ hepatomegaly in different schools, but less than $0.5 \%$ hepatosplenomegaly), there was some evidence for a persistent reduction in hepatomegaly: and separate snail studies provided evidence for a reduction in transmission (Sturrock et al., in preparation).

The simple conclusion, therefore, was that treatment of 
schoolchildren only did have a marked and persistent impact, not only on intensity of infection and morbidity among the treated target population, but also on transmission in the community as a whole. The effect was in fact better in Area $\mathrm{S}$ than in Area $\mathrm{H}$ : and the procedure was considerably less costly, in terms of both drug usage and the time taken for diagnosis and delivery by a skilled technical team.

In areas of relatively low morbidity, such as Kangundo, retreatment of schooldchildren need not be carried out for two, or even three, years: this is in contrast to high morbidity areas, in which retreatment may be required at more frequent intervals (below). Plans are now in progress in Kenya for the development of a large-scale national control programme based on chemotherapy of schoolchildren. However, it can still be argued that is not ideal in the long-term, for several reasons. First, the costs, not only of drugs but also of diagnosis and delivery, are still high, even though they are less than those involved in treating whole communities. In addition, there is still a need for repeated surveillance and retreatment, all of which has to be based on vertically-orientated and disease-specific national control programmes, which are difficult to maintain and fund. Finally, the serious possibility must be considered of the emergence of drug-resistant parasites, which has certainly occurred with at least one anti-schistosome drug, oxamniquine. In the long run, therefore, prevention is still better than cure, and new methods of control, in particular vaccination, should still be sought.

\section{PROGRESS TOWARDS VACCINATION:} EVIDENCE FOR IMMUNITY IN MAN

Studies in experimental animals have provided clear evidence for the development of immunity to infection after appropriate immunisation, and several groups have cloned and expressed a range of candidate vaccine antigens that are protective in these experimental models (reviewed by Dissous et al., 1987). However, one of the problems about these models is that each one differs quite markedly from the next, in both the extent and more importantly the mechanisms of protective immunity, and none of them can be taken a priori as predictors of what will happen in man: complementary studies on immunity in man are therefore essential.

The probable development with age of an acquired immunity to reinfection in man is reflected in the observation, in all the study areas described above, of a very strong age dependence of reinfection. After chemotherapy, reinfection occurred predominantly among the young schoolchildren, and was markedly less in older children and adults. Clearly, this lack of reinfection among the older age groups could be due either to an acquired immunity, or to a lack of exposure, or to both, and the only way of distinguis- hing between these two possibilities is to examine changes in exposure by age, by repeated observations of levels of water contact.

Extensive water contact observations have been made in all of the study areas, but in particular in the village of Iietune (Butterworth et al., 1984, 1988). In this area, treatment had been offered to all infected individuals in October 1983, at that stage without the inclusion of a surrounding buffer zone. Transmission therefore occurred over the following nine months, and extensive reinfection occurred among the younger children, peaking in those aged 9 to 12 years, with a sharp decline in older children and adults. In contrast, total duration of water contact showed a much later peak between the ages of 16 and 24 and continued throughout adult life, although with some decline. However, a possible explanation for this divergence in age distribution was that adults might have a different and less infective type of water contact. When the water contact data were weighted to give more emphasis, among other things, to activities such as swimming and bathing that are carried out mainly by the younger children and are more likely to lead to infection, then it was found that the distribution by age now more closely approximated (and was significantly correlated, on an individual basis) with the distribution of reinfection. However, the declining levels of exposure with age were still insufficient to account for the decline in reinfection: young adults aged 20 to 24, for example, had over half the levels of weighted exposure that were observed in the 9 to 12 year olds, but only $1 \%$ of the levels of reinfection.

We conclude that, although levels of exposure do decline in older children and adults, this is not a sufficient explanation for their lack of reinfection after treatment. Instead, there would appear to be the development, around the age of puberty, of an acquired resistance that has been separately found to be dependent on previous experience of infection and that is of major importance in limiting reinfection in older individuals. Similar conclusions have been reached by Wilkins et al. (1987) in a series of elegant and detailed studies on S. haematobium infections in The Gambia.

The next question is whether any immunological correlates can be found that might help to explain this very slow development of immunity with age. One approach to this question is to look at responses to individual antigens that have been shown, for example in animal experiments, to be associated with immunity: a second is to take a slightly more holistic approach, treating the organism as a whole, and working on the hypothesis that the net level of immunity is going to derive from a complex mixture of different responses to a range of different parasite antigens.

Taking the latter approach first, we had previously studied a cohort of 129 Iietune schoolchildren, aged 9 to 16 , 
who had been treated and followed for reinfection over a 21 month period, and this had provided the first indication of the slow development with age of an acquired resistance to reinfection that was independent of changes in exposure in this limited age range (Butterworth et al., 1985). Evidence was obtained that the reason why immunity takes so long to develop in these children is not that the younger children fail to mount a protective response, but rather that they also produce inappropriate antibodies that block the expression of the protective response (Butterworth et al., 1987; Dunne et al., 1988). These blocking antibodies are elicted mainly in response to egg polysaccharide antigens: they cross-react with carbohydrate epitopes expressed at the schistosomulum surface: they comprise both IgM and IgG2 antibodies: and they slowly decline with age, permitting the expression of the protective responses and hence of immunity. The nature of the protective responses that are blocked is still unclear, although the implication is that they are also primarily directed against schistosomulum surface carbohydrates.

The alternative approach is to examine responses to individual antigens, one example being the nature of lymphocyte responses to the antigen $\mathrm{Sm} 28$. This is a glutathioneS-transferase that has been studied in detail by Capron and colleagues (Balloul et al., 1987): it is a parenchymal enzyme that is released and transiently expressed on the surface of the schistosomulum, where it may form the target for eosinophil-mediated ADDC attack. It has been cloned and expressed in E. coli and yeast, and the recombinant molecule is highly protective in rats and mice. Preliminary protection experiments in baboons have been promising, and it is one of the best candidate vaccine antigens out of several that are now available. In initial experiments, it was found that two thirds of a group of 40 patients of all ages showed lymphocyte proliferative responses to Sm28. More recently, I. Wolowczuk and K. Gachuhi in Nairobi have examined individual responses to p28 in detail, and have found that they include reactions against several different peptides derived from the amino acid sequence, with those against the $\mathrm{C}$-terminal end being most frequent among different patients. The relationship of these responses to resistance to reinfection after treatment is not yet known, and this work is descriptive at the moment; but it is a long-term approach to examining the role of responses to individual antigens in mediating protection. The important point is that human responses do not appear to be as severely restricted as they are, for example, against malaria antigens. In summary, candidate vaccine antigens are becoming available, and the evidence so far from human field studies suggests that they could eventually be used in man.

\section{DETERMINANTS OF MORBIDITY}

A further problem in human field studies concerns the various factors that determine the expression of severe disease. The aim of control is to reduce morbidity, and morbidity is at least partly dependent on intensity of infection. However, intensity may not be the only factor. In our original study areas around Kangundo, we had found high prevalence and intensities of infection, but surprisingly little evidence of severe morbidity. In contrast, we have recently identified an area near Kambu, in the eastern part of Machakos District, in which there is evidence of extremely severe morbidity: in one school, $16 \%$ had hepatomegaly, but an additional $17 \%$ had hepatosplenomegaly. In many cases this condition was gross, and was associated with classical portal hypertension and oesophageal varices. Formal comparison of schools in Kangundo and Kambu revealed that hepatomegaly was related to intensity of infection, and was little different between the two areas (Fulford et al., in preparation). In contrast, splenomegaly associated with hepatomegaly was almost undetectable in Kangundo: but in Kambu it was highly prevalent and again related to intensity of schistosome infection. In Kambu, malaria was also a contributory factor, but was relatively minor and could be distinguished from the schistosomeinduced morbidity.

An extensive study is being undertaken to determine the reason for the difference between the two areas, the possibilities including:

1) Host genetic differences. These are unlikely to account for the overall differences between the areas, since all the people are of the relatively homogeneous Akamba tribe, but they might contribute to individual differences within the high morbidity area.

2) Parasite strain differences. Strains have been established from both areas, but have so far shown no biological differences.

3) The nature of exposure, and in particular the age at first infection, in that the small liver of a young child may be more severely damaged by an initial heavy egg load. So far, however, the distribution of age-intensity curves for the communities in Kambu and Kangundo appear similar.

4) Interactions with other infectious diseases, in particular malaria, hepatitis of different types, and leishmaniasis. It is already known that the hepatosplenomegaly in Kambu is not directly attributable to malaria, but this does not exclude the possibility that previous or repeated malaria infections might contribute to or exacerbate schistosome-induced pathology.

5) Nutritional status. Overall nutrition is worse in Kambu than in Kangundo, and in both areas those with severe morbidity are less well nourished than those without. So far, however, cross-sectional surveys, although limited, support the hypothesis that the nutritional defect in severely sick children is a consequence rather than a cause of their severe morbidity.

6) Maternal infection status, in particular in relation to 
the development in the young infant of various immunoregulatory mechanisms, especially the anti-idiotypic $\mathrm{T}$ cells clearly described by Colley and colleagues from Brazil (reviewed by Colley et al., 1989).

Complete resolution of these possible explanations has not yet been achieved: but the problem is important, since the area of Kambu is typical of much of Kenya, and indeed or rural Africa in general. It is a harsh environment, dry, with multiple diseases and apparently poor nutrition, recently settled by people who have been pushed by population pressure out of the older, more fertile regions: and control measures that are useful in a conventional area of low morbidity may be much less effective here, especially if intensity of infection is not the only determinant of disease.

\section{CONCLUSIONS}

During recent years, work by many groups has led to advances both in the delivery of chemotherapy as an established control measure and in the acquisition of some basic information and materials that will be required for the development of a vaccine. However, there are still many questions that remain to be tackled through detailed field studies. For example, we need to know to what extent the expression of immunity in man is multifactorial, and what various immune responses correlate positively with the expression of immunity in older individuals. We also need to know much more about the impact of chemotherapy itself, both on the expression of immunity and on the development or recurrence of morbidity after treatment: and we still do not know enough about the factors that determine the expression of severe disease. Finally, if a vaccine that can be used in man does become available, we shall need to know what happens if an incompletely-protected person develops a subsequent low level infection: will he lose his immunity, for example, because he develops blocking antibodies, or will he show increased or reduced pathology? These are all difficult, and perhaps impossible, questions to answer, but they should be posed.
Acknowledgments. - Work by the author and his colleagues summarised in this review was suppported by grants from the Edna McConnel, Clark Foundation, the Medical Research Council, the European Commission, and the Rockefeller Foundation/World Health Organisation Joint Funding Venture.

\section{REFERENCES}

Anderson R. M. : Determinants of infection in human schistosomiasis. In: Mahmoud A. A. F. (ed.), Baillière's Clinical Tropical Medicine and Communicable Diseases, Vol. 2, No. 2. Baillière/Tindall London, 1987, 279-300.

Balloul J. M., Sondermeyer P., Dreyer D. et al. : Molecular cloning of a protective antigen of schistosomes. Nature (London), 1987, 326, 149-153.

Butterworth A. E., Dalton P. R., Dunne D. W. et al. : Immunity after treatment of human schistosomiasis mansoni. I. Study design, pretreatment observations and the results of treatment. Trans. Roy. Soc. Trop. Med. Hyg., 1984, 78, 108-123.

Butterworth A. E., Capron M., Cordingley J. S. et al. : Immunity after treatment of human schistosomiasis mansoni. II. Identification of resistant individuals, and analysis of their immune responses. Trans. Roy. Soc. Trop. Med. Hyg., 1985, 79, 393-408.

Butterworth A. E., Bensted-Smith R., Capron A. et al : Immunity in human schistosomiasis: prevention by blocking antibodies of the expression of immunity in young children. Parasitology, 1987, 94, 281-300.

Butterworth A. E., Fulford A. J. C., Dunne D. W. et al. : Longitudinal studies on human schistosomiasis. Phil. Trans. Roy. Soc. Lond. B, 1988, 321, 495-511.

Butterworth A. E., Corbett E. L., Dunne D. W. et al. : In: McAdam K. P. W. J. (ed.), New Strategies in Parasitology. Churchill Livingstone, Edinburgh, 1989, 193-214.

Colley D. G., Montesano M. A., Eloi-Santos S. M. et al. : Idiotypic networks in schistosomiasis. In: McAdam K. P. W. J. (ed.), New Strategies in Parasitology. Churchill Livingstone, Edinburgh, 1989, 179-182.

Dissous C., Balloul J. M., Pierce R., Capron A. : Protective antigens of schistosomes. In: Mahmoud A. A. F. (ed.), Baillière's Clinical Tropical Medicine and Communicable Diseases, Vol. 2, No 2. Baillière/Tindall, London, 1987, 267-277.

Dunne D. W., Grabowska A. M., Fulford A. J. C. et al. : Human antibody responses to Schistosoma mansoni: the influence of epitopes shared between different life-cycle stages on the response to the schistosomulum. Eur. J. Immunol., 1988, 18, 123-131.

Wilkins H. A., Blumenthal U. J., Hagan P. et al. : Resistance to reinfection after treatment of urinary schistosomiasis. Trans. Roy. Soc. Trop. Med. Hyg., 1987, 81, 29-35. 